INPLASY

PROTOCOL

To cite: Li et al. Combination of Acupuncture and Chinese Herbal Medicine for Eczema: A Protocol for Systemic Review and Meta-Analysis. Inplasy protocol 2021110026. doi: 10.37766/inplasy2021.11.0026

Received: 08 November 2021

Published: 08 November 2021

Corresponding author: Lin Du

dulin683@126.com

Author Affiliation:

Dongzhimen Hospital, Beijing University of Chinese

Medicine.

Support: None.

Review Stage at time of this submission: Preliminary searches.

Conflicts of interest:

None declared.

\section{Combination of Acupuncture and Chinese Herbal Medicine for Eczema: A Protocol for Systemic Review and Meta-Analysis}

Li, WX1; Yao, ZH²; Sun, HP3; Du, L4.

Review question / Objective: The aim of the study is to evaluate the efficacy and safety of combination of acupuncture and Chinese herbal medicine in treating eczema. Condition being studied: Eczema is a complex chronic inflammatory disease of the skin charaterized by itching, disrupted epidermal barrier function. It has a huge negative influence on patients' life. In recent years, there are many randomized controlled trials that show that combination of acupuncture and Chinese herbal medicine plays an important role in treating eczema. And no prior revelant systemic evaluation has bee found. Therefore, we conducted a systemic review and meta-analysis to evaluate the efficacy and safety of combination of acupuncture and Chinese herbal medicine in treating eczema.

INPLASY registration number: This protocol was registered with the International Platform of Registered Systematic Review and Meta-Analysis Protocols (INPLASY) on 08 November 2021 and was last updated on 08 November 2021 (registration number INPLASY2021110026).

\section{INTRODUCTION}

Review question / Objective: The aim of the study is to evaluate the efficacy and safety of combination of acupuncture and Chinese herbal medicine in treating eczema.
Condition being studied: Eczema is a complex chronic inflammatory disease of the skin charaterized by itching, disrupted epidermal barrier function. It has a huge negative influence on patients' life. In recent years, there are many randomized controlled trials that show that combination 
of acupuncture and Chinese herbal medicine plays an important role in treating eczema. And no prior revelant systemic evaluation has bee found. Therefore, we conducted a systemic review and metaanalysis to evaluate the efficacy and safety of combination of acupuncture and Chinese herbal medicine in treating eczema.

\section{METHODS}

Participant or population: Patients with eczema.

Intervention: Combination of acupuncture and Chinese herbal medicine.

Comparator: Western medicine.

Study designs to be included: Randomized controlled trials.

Eligibility criteria: Inclusion Criteria:(1) Patients diagnosed with eczema. Age, gender or race is not taken into account;(2) The treatment group receives combination of acunpuncture (including classical acupuncture, fi re needle, eletrcoacupuncture, collateral-pricking and bloodletting, but excluding laser acupuncture, acupoint injection, catgut embedding are excluded ) and Chinese herbal medicine while the control group receives western medicine;(3) Outcomes includes at least one of the following measures: clinical effective rate, scoring atopic dermatitis index (SCORAD),eczema area and severity index score (EASI),itching visual analogue score (VAS); dermatology life quality index (DLQI) and adverse effects.(4) Randomized controlled trials only. Exclusion Criteria:(1) full text cannot be obtained or data cannot be extracted after contracting original authors;(2) duplicated data.

Information sources: PubMed, Embase, Cochrane, China National Knowledge Infrastructure(CNKI), Wanfang Database, and Chinese Science and Technology Periodical Database (VIP).
Main outcome(s): clinical effective rate, scoring atopic dermatitis index (SCORAD),eczema area and severity index score (EASI), and itching visual analogue score (VAS).

Additional outcome(s): Dermatology life quality index (DLQI) and adverse effects.

Quality assessment / Risk of bias analysis: The methodological quality of the eligible RCTs was evaluated independently by two researchers (Zihan Yao, Huapeng Sun) according to the Cochrane Handbook for Systematic Reviews of Interventions. The details included: random sequence generation, allocation concealment, blinding of participants and personnel, blinding of outcome assessors, incomplete outcome data, selective reporting and other bias. The risk of bias for each item was summarized into three categories: low, unclear, and high. Any differences encountered were resolved by a third researcher (Lin Du).

Strategy of data synthesis: All data will be conducted by RevMan version 5.3. Dichotomous data will be reported as relative ratio (RR) whereas continuous data as mean difference (MD). Both of them corresponding $95 \%$ confidence interval (Cl) will be calculated. Heterogeneity will be assessed using the 12 . $12<50 \%$ indicats that the studies have homogeneity, so fixed effects model will be applied, otherwise the random effects model will be applied. We will perform meta-analyses for trials if the design, participants, interventions, control, and outcome measures of studies are similar. Bulk data will be synthesized quantitatively by descriptive counting. If the data are not available for quantitative analysis, we will report result by qualitative description.

Subgroup analysis: If there is significant heterogeneity between the study results, subgroup analysis will be performed to investigate differences in gender, age and types of acupuncture.

Sensitivity analysis: Sensitivity analysis will be used to find the sources of 
heterogeneity and item by item will be used.

Language: Chinese and English.

Country(ies) involved: China.

Keywords: acpuncture; eczema; Chinese herbal medicine; systemic review; metaanalysis.

Contributions of each author:

Author 1 - Weixin Li.

Email: 751111371@qq.com

Author 2 - Zihan Yao.

Author 3 - Huapeng Sun.

Author 4 - Lin Du.

Email: dulin683@126.com 